\title{
Epidemiología del paciente pediátrico quemadio en el Hospital Baca Ortiz, Quito, Ecuador
}

\section{Epidemiology of the burned pediatric patient at Baca Ortiz Hospital, Quito, Ecuador}

Pablo GALLEGOS TORRES*, Thalía ARGÜELLO GORDILLO** Regina REAL FLORES ${ }^{\star \star *}$, Olimpia TRUJILLO ORBE ${ }^{\star \star \star *}$

\section{Resumen}

Introducción y objetivo. Las quemaduras son un problema mundial de salud pública por su gran impacto físico, psicológico, económico y social.

El objetivo de este trabajo es describir las características epidemiológicas de los pacientes pediátricos con quemaduras en nuestro entorno asistencial.

Material y método. Realizamos un estudio descriptivo retrospectivo tomando como universo al total de pacientes pediátricos con quemaduras atendidos en el Hospital Baca Ortiz de Quito, Ecuador, de enero a diciembre del 2016.

Resultados. Recogimos un total de 343 pacientes pediátricos con quemaduras de los que 180 requirieron hospitalización (52.48\%), con predominio de menores de 5 años (67.6\%). El género masculino fue el más afectado $(55.7 \%)$ y las quemaduras por líquido caliente representaron el 70.6\%. Las de mayor estancia hospitalaria fueron las quemaduras eléctricas. Registramos una mortalidad del $1.1 \%$ relacionada con quemaduras por fuego, de más del $50 \%$ de superficie corporal y con compromiso de vía aérea.

Conclusiones. Los menores de 5 años son más vulnerables para sufrir quemaduras. La principal etiología fue líquido hirviente. Los casos fatales tuvieron relación con quemaduras por fuego y lesión de vía aérea. Las quemaduras eléctricas son devastadoras, la severidad de sus lesiones ocasiona mayor estancia hospitalaria y un aumento de la necesidad de amputación de la extremidad afectada.

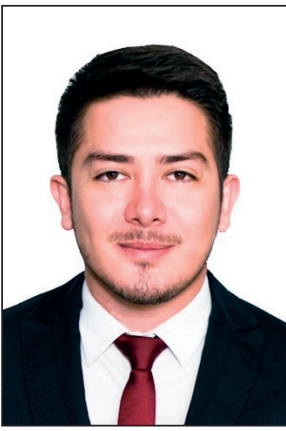

Gallegos Torres P.

Abstract

Background and objective. Burns are considered a public health problem worldwide due to the great physical, psychological, economic and social impact they cause.

The objective of this paper is to describe epidemiological characteristics of pediatric patients with burns in our regional area.

Methods. A retrospective descriptive study was carried out taking as universe total number of pediatric patients with burns treated at Baca Ortiz Hospital in Quito, Ecuador, from January to December 2016.

Results. We studied 343 pediatric patients with burns of which 180 required hospitalization $(52.48 \%)$. Predominance of younger than 5 years old $(67.6 \%)$ was observed. Masculine gender was most affected $(55.7 \%)$, burns caused by hot liquid reported a frequency of $70.6 \%$. Electric burns reported longer hospital stay. Mortality of $1.1 \%$ was registered related to burns caused by fire, greater than $50 \%$ of body surface area and airway commitment.

Conclusions. Children younger than 5 years old are most vulnerable to burn. Hot liquid was the main etiology of burns. Fatal cases were related to burns caused by fire and airway injury. Electric burns are devastating; severity of injuries causes longer hospital stay and greater need for amputation of affected limb.
Palabras clave Quemaduras, Quemaduras pediátricas, Epidemiología quemados.
4b Diagnóstico
18 marzo/2019
6 mayo/2019

\section{Recibido [esta versión]}
Aceptado

Conflicto de intereses: Los autores declaran no tener ningún interés financiero relacionado con el contenido de este artículo.

Financiación: No hubo fuentes externas de financiación para este trabajo.

\begin{tabular}{|lr|}
\hline Key words & $\begin{array}{l}\text { Burns, Pediatrics burns, } \\
\text { Burns epidemiology. }\end{array}$ \\
& \\
Level of evidence & $4 \mathrm{~b}$ Diagnostic \\
Received [this version] & $18 \mathrm{March} / 2019$ \\
Accepted & $6 \mathrm{May} / 2019$ \\
\hline
\end{tabular}

\footnotetext{
* Médico Residente de Cirugía Plástica, Hospital Baca Ortiz, Quito, Ecuador

** Médico Cirujano, Pontificia Universidad Católica del Ecuador, Quito, Ecuador

*** Pediatra, Hospital Baca Ortiz, Quito, Ecuador

${ }^{* * \star \star}$ Cirujano Plástico, Hospital Baca Ortiz, Quito, Ecuador
} 
Introducción

Las quemaduras constituyen un problema de salud pública a nivel mundial; según datos de la Organización Mundial de la Salud (OMS) provocan alrededor de 180.000 muertes al año. La mayoría de casos fatales ocurren en países de bajos y medianos ingresos, registrando más del $95 \%$ del total de las muertes por esta causa. ${ }^{(1)}$ La población infantil es más vulnerable a las quemaduras debido a que su capacidad física, mental y su juicio no son apropiados para reaccionar rápida y adecuadamente ante algunos factores de riesgo presentes en el medio ambiente,,$^{(2)}$ es por eso que las quemaduras son las lesiones prevenibles más frecuentes en menores de 5 años de edad. ${ }^{(3)}$ Las estadísticas muestran que entre el $80 \%$ y el $90 \%$ de las quemaduras se produce en el hogar. ${ }^{(1)}$ Se estima que el riesgo de muerte por quemaduras en la niñez es elevado, con una tasa mundial de 2.5 defunciones por cada 100.000 habitantes. ${ }^{(4)}$

Las quemaduras producen lesiones en el órgano más grande del cuerpo humano: la piel. Entre las complicaciones agudas que contribuyen a la mortalidad temprana encontramos la insuficiencia pulmonar, la insuficiencia renal aguda y el choque del paciente quemado, que generalmente surgen dentro de las primeras 72 horas tras la quemadura. ${ }^{(5)}$ Como complicaciones tardías que aumentan la mortalidad en estos pacientes encontramos las infecciones de partes blandas, la sepsis y el fallo multiorgánico secundario. ${ }^{(5)}$ Las quemaduras no fatales son una de las principales causas de morbilidad, que incluye hospitalización prolongada, desfiguración y discapacidad que a su vez suelen generar estigmatización y rechazo. ${ }^{(1)}$

Todo esto lo convierte en un problema de salud pública debido al impacto físico, psicológico, económico y social que ocasiona a los pacientes y a sus familiares, por lo que se busca comprender la etiología de este tipo de lesiones para desarrollar estrategias de prevención efectivas.

El objetivo del presente trabajo es describir las características epidemiológicas de los pacientes pediátricos que acudieron con quemaduras al Hospital Baca Ortiz (HBO) de la ciudad de Quito, Ecuador, tomado como periodo de estudio desde enero a diciembre del 2016, para poder hacer una estimación de la incidencia y características más relevantes de este tipo de lesiones entre la población pediátrica de nuestro entorno.

\section{Material y método}

Realizamos un estudio descriptivo retrospectivo tomando como universo el total de pacientes pediátricos con quemaduras atendidos en el HBO de enero a diciembre del 2016.
Consideramos las siguientes variables: edad, género, etiología de la quemadura, extensión, profundidad y localización de las lesiones para la clasificación de su gravedad, tratamiento quirúrgico requerido, días de hospitalización y mortalidad.

Clasificamos los pacientes en una de las tres categorías propuestas por la American Burn Association (ABA) (Asociación Americana de Quemaduras) según la gravedad de sus lesiones: ${ }^{(6)}$ quemadura leve, moderada y grave.

Los datos fueron ingresados en Microsoft Excel y analizados en el programa SPSS para Windows (Versión 23) mediante frecuencias absolutas, frecuencias relativas y medias.

\section{Resultados}

Durante los meses de estudio registramos un total de 343 pacientes pediátricos con quemaduras que recibieron atención médica en el HBO, de los cuales 180 $(52.48 \%)$ requirieron hospitalización, mientras que los 163 restantes $(47.52 \%)$ fueron tratados de manera ambulatoria. Observamos que 191 pacientes (55.7\%) fueron de género masculino y 152 (44.3\%) femenino, con una edad media de 4.4 años (máxima de 14 años y mínima de 8 días). En el Gráfico 1 presentamos la distribución de los pacientes según grupo etário y género, con un predominio de 131 pacientes (38.2\%) en edad pre-escolar y un porcentaje acumulado del $67.6 \%$ correspondiente a menores de 5 años.

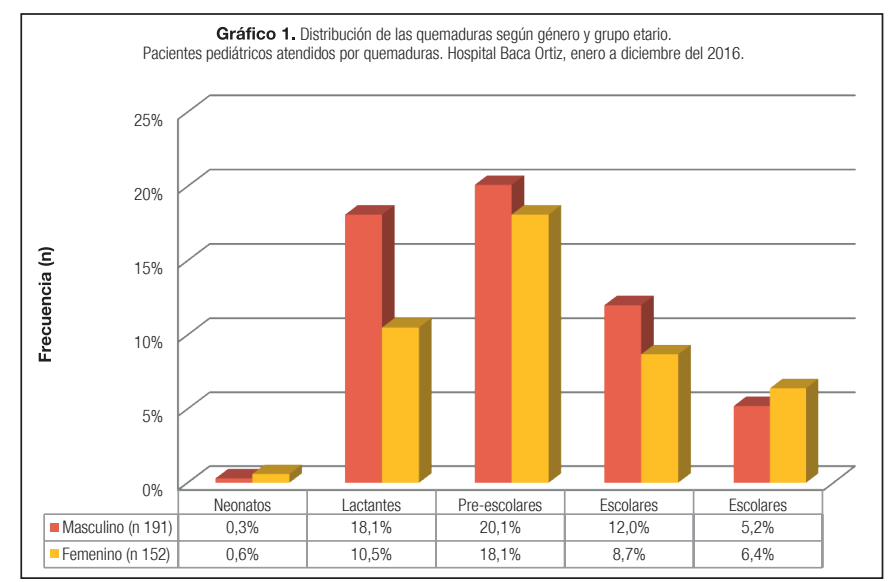

Según la etiología de las lesiones, 242 pacientes (70.6\%) correspondieron a lesiones causadas por líquido caliente, mientras que las lesiones menos frecuentes fueron aquellas causadas por electricidad, con 16 pacientes (4.7\%) (Gráfico 2).

Dentro del grupo de 180 pacientes hospitalizados clasificamos la gravedad de sus lesiones según los criterios de la ABA, encontrando 35 pacientes (19.4\%) con lesiones leves, $28(15.6 \%)$ con lesiones moderadas y 117 (65\%) con quemaduras graves (Gráfico 3). 

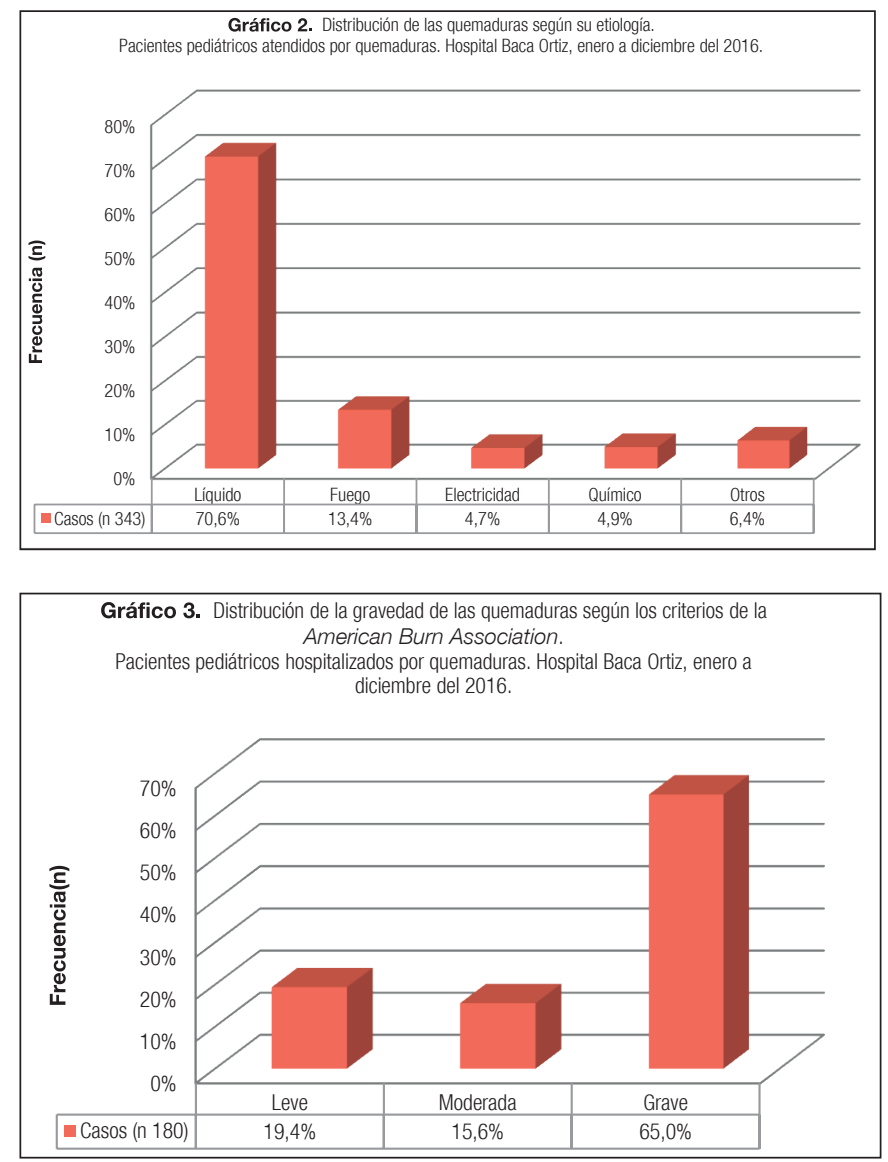

De los pacientes clasificados con lesiones graves, 7 (6\%) requirieron tratamiento en la Unidad de Cuidados Intensivos del hospital, con una estancia promedio de 13.4 días.

De los pacientes hospitalizados, 75 (41.7\%) sufrieron quemaduras en 1 zona corporal; el resto tuvieron compromiso de múltiples regiones, llegando a presentar 4 pacientes $(2.2 \%)$ compromiso de hasta 5 zonas corporales (Gráfico 4).

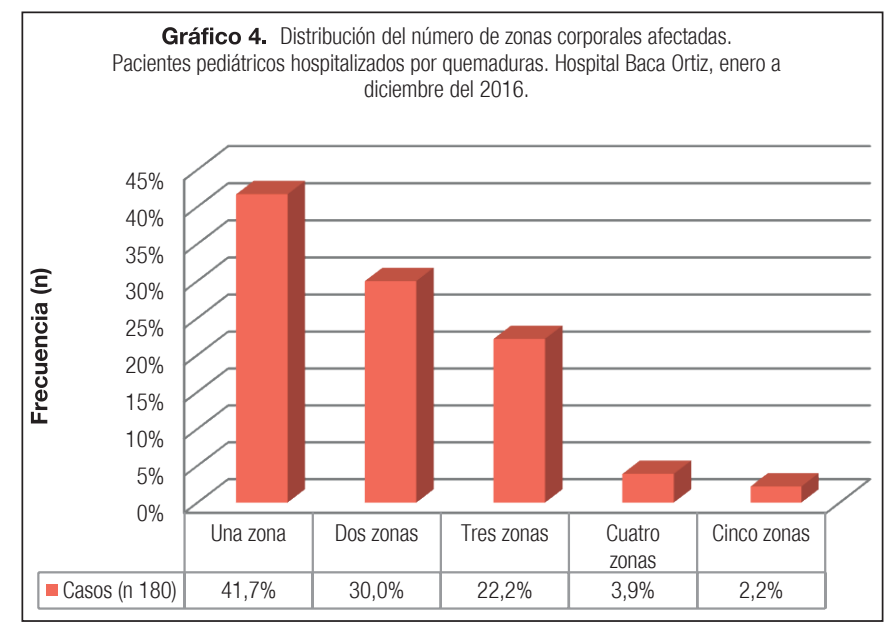

Entre los pacientes hospitalizados, la región corporal más afectada fue tórax/abdomen, que registró un total de 97 casos (53.9\%). En menor proporción se presentaron pacientes con lesiones que representan por si solas criterios de gravedad, independientemente de su profundidad o extensión, tales como quemaduras que comprometen cabeza, cuello, manos, pies, genitales y vía aérea (Gráfico 5).

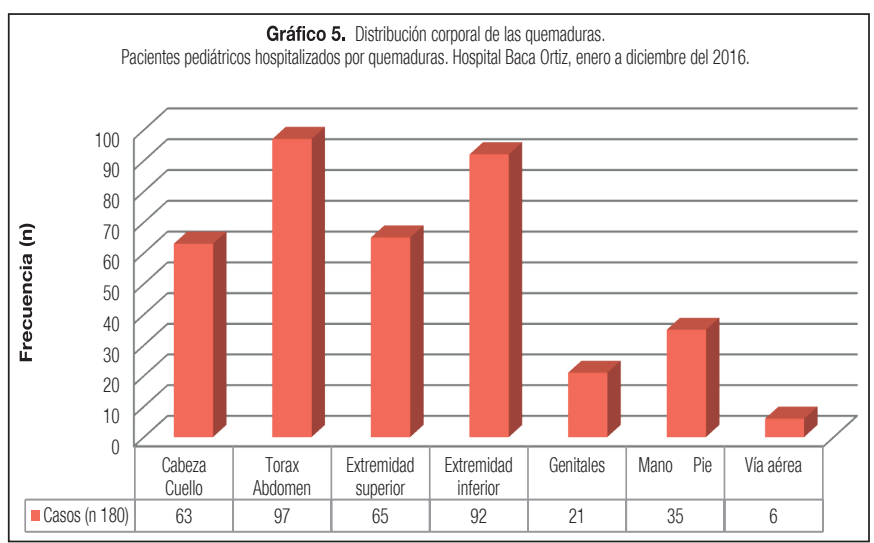

Nota: para una correcta interpretación del Gráfico 5, se debe tener en cuenta que de los 180 pacientes hospitalizados por quemaduras, 105 (58.3\%) presentaron compromiso de múltiples zonas corporales.

Tomando en cuenta que los pacientes hospitalizados son sometidos a limpiezas quirúrgicas cada 48 horas, observamos que la necesidad de otros tipos de tratamiento quirúrgico se presentó en 63 pacientes (35\%), de los cuales en 59 casos $(32.8 \%)$ se realizó cobertura con injerto cutáneo y en $4(2.2 \%)$ amputación de la extremidad afectada (Gráfico 6). Dentro de este último grupo, 3 casos $(75 \%)$ correspondieron a quemaduras eléctricas y 1 $(25 \%)$ a quemadura por fuego.

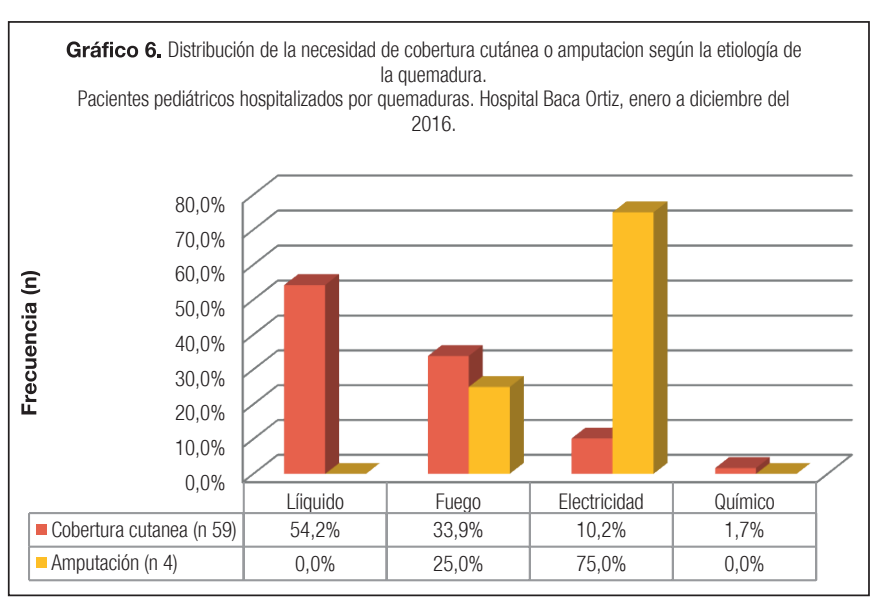

La estancia hospitalaria fue de 19.4 días como media (mínima de 1 día y máxima de 111 días). El 31.6\% de los pacientes con lesiones leves tuvo una estancia hospitalaria superior a 15 días, en contraste con el 50\% con lesiones moderadas y el $53 \%$ con lesiones graves (Tabla I).

Registramos el fallecimiento de 2 pacientes (1.1\%) clasificados dentro del grupo de quemaduras graves; ambos eran de género femenino, con quemaduras ocasionadas por fuego, compromiso de vía aérea y una superficie corporal afectada mayor del $50 \%$. 
Tabla I. Estancia hospitalaria según la superficie corporal quemada, etiología y gravedad de las quemaduras. Pacientes pediátricos hospitalizados por quemaduras. Hospital Baca Ortiz, enero a diciembre del 2016.

\begin{tabular}{|c|c|c|c|c|c|c|c|c|c|c|c|}
\hline $\begin{array}{c}\text { ESTANCIA } \\
\text { HOSPITALARIA }\end{array}$ & \multicolumn{3}{|c|}{ SUPERFICIE CORPORAL QUEMADA } & \multicolumn{5}{|c|}{ ETIOLOGÍA DE LAS QUEMADURAS } & \multicolumn{3}{|c|}{ GRAVEDAD DE LAS QUEMADURAS } \\
\hline 1-15 días & $\begin{array}{c}83 \\
(61.9 \%)\end{array}$ & $\begin{array}{c}10 \\
(29.4 \%)\end{array}$ & $\begin{array}{c}0 \\
(0 \%)\end{array}$ & $\begin{array}{c}63 \\
(55.3 \%)\end{array}$ & $\begin{array}{c}12 \\
(32.4 \%)\end{array}$ & $\begin{array}{c}5 \\
(41.7 \%)\end{array}$ & $\begin{array}{c}12 \\
(80 \%)\end{array}$ & $\begin{array}{c}1 \\
(50 \%)\end{array}$ & $\begin{array}{c}24 \\
(68.4 \%)\end{array}$ & $\begin{array}{c}14 \\
(50 \%)\end{array}$ & $\begin{array}{c}55 \\
(47 \%)\end{array}$ \\
\hline 16-30 días & $\begin{array}{c}34 \\
(25.4 \%)\end{array}$ & $\begin{array}{c}10 \\
(29.4 \%)\end{array}$ & $\begin{array}{c}3 \\
(25 \%)\end{array}$ & $\begin{array}{c}33 \\
(28.9 \%)\end{array}$ & $\begin{array}{c}9 \\
(24.3 \%)\end{array}$ & $\begin{array}{c}1 \\
(8.3 \%)\end{array}$ & $\begin{array}{c}3 \\
(20 \%)\end{array}$ & $\begin{array}{c}1 \\
(50 \%)\end{array}$ & $\begin{array}{c}8 \\
(22.9 \%)\end{array}$ & $\begin{array}{c}6 \\
(21.4 \%)\end{array}$ & $\begin{array}{c}33 \\
(28.2 \%)\end{array}$ \\
\hline 31-45 días & $\begin{array}{c}13 \\
(9.7 \%)\end{array}$ & $\begin{array}{c}10 \\
(29.4 \%)\end{array}$ & $\begin{array}{c}4 \\
(33.3 \%)\end{array}$ & $\begin{array}{c}14 \\
(12.3 \%)\end{array}$ & $\begin{array}{c}11 \\
(29.8 \%)\end{array}$ & $\begin{array}{c}2 \\
(16.7 \%)\end{array}$ & $\begin{array}{c}0 \\
(0 \%)\end{array}$ & $\begin{array}{c}0 \\
(0 \%)\end{array}$ & $\begin{array}{c}3 \\
(8.7 \%)\end{array}$ & $\begin{array}{c}5 \\
(17.9 \%)\end{array}$ & $\begin{array}{c}19 \\
(16.3 \%)\end{array}$ \\
\hline 46-60 días & $\begin{array}{c}3 \\
(2.3 \%)\end{array}$ & $\begin{array}{c}3 \\
(8.9 \%)\end{array}$ & $\begin{array}{c}4 \\
(33.3 \%)\end{array}$ & $\begin{array}{c}4 \\
(3.5 \%)\end{array}$ & $\begin{array}{c}3 \\
(8.1 \%)\end{array}$ & $\begin{array}{c}3 \\
(25 \%)\end{array}$ & $\begin{array}{c}0 \\
(0 \%)\end{array}$ & $\begin{array}{c}0 \\
(0 \%)\end{array}$ & $\begin{array}{c}0 \\
(0 \%)\end{array}$ & $\begin{array}{c}2 \\
(7.1 \%)\end{array}$ & $\begin{array}{c}8 \\
(6.8 \%)\end{array}$ \\
\hline$>61$ días & $\begin{array}{c}1 \\
(0.7 \%)\end{array}$ & $\begin{array}{c}1 \\
(2.9 \%)\end{array}$ & $\begin{array}{c}1 \\
(8.4 \%)\end{array}$ & $\begin{array}{c}0 \\
(0 \%)\end{array}$ & $\begin{array}{c}2 \\
(5.4 \%)\end{array}$ & $\begin{array}{c}1 \\
(8.3 \%)\end{array}$ & $\begin{array}{c}0 \\
(0 \%)\end{array}$ & $\begin{array}{c}0 \\
(0 \%)\end{array}$ & $\begin{array}{c}0 \\
(0 \%)\end{array}$ & $\begin{array}{c}1 \\
(3.6 \%)\end{array}$ & $\begin{array}{c}2 \\
(1.7 \%)\end{array}$ \\
\hline
\end{tabular}

\section{Discusión}

Las quemaduras son una de las lesiones más serias que pueden afectar al ser humano. Al ser eventos prevenibles, el conocimiento de las características epidemiológicas del paciente quemado es de vital importancia para diseñar e implementar intervenciones efectivas utilizando métodos basados en la educación a la comunidad con el fin de salvaguardar a la población.

Planteamos un estudio de tipo retrospectivo en el que las variables utilizadas fueron únicamente las disponibles en los registros de la Unidad de Quemados del Hospital Baca Ortiz; además, al tratarse de un estudio descriptivo, los datos de dichos registros pueden estar incompletos o sesgados. Este estudio se realizó en un hospital de tercer nivel considerado como el principal centro de referencia a nivel nacional, el cual recibe pacientes de todo el centro y norte del Ecuador continental.

Observamos un predominio de pacientes de género masculino a razón de 1.3:1 con respecto al género femenino, datos similares a los obtenidos en estudios de la región llevados a cabo en Chile, Colombia, Ecuador y México ${ }^{(7-14)}$ y en otras partes del mundo: África, China, Estados Unidos, Irán, Israel y Turquía. ${ }^{(13-16)}$ Esta relación se invierte en el grupo de adolescentes a razón de $0.8: 1$, lo cual puede atribuirse a factores culturales y socioeconómicos que implican una temprana intervención de la mujer como responsable del hogar, llevándola a manipular artefactos de cocina con el riesgo que esto implica. ${ }^{(11)}$

Coincidiendo con estudios similares, ${ }^{(7-14)}$ los menores de 5 años $(67.6 \%)$ son la población más vulnerable para sufrir este tipo de lesiones.

En relación al agente causal, las quemaduras más frecuentes se debieron al contacto con líquido caliente (70.6\%), seguidas por las producidas por exposición a fuego y electricidad, resultados similares a los descritos por otros autores. . $^{(7,8,10,12,13)}$

Con respecto a la zona corporal más afectada encontramos una frecuencia del $53.9 \%$ correspondiente a tórax/abdomen, similar a lo reportado en publicaciones previas, ${ }^{(10,17)}$ en tanto que las quemaduras en genitales fueron las menos frecuentes $11.7 \%$.

La estancia hospitalaria en pacientes con quemaduras categorizadas como moderadas o graves fue mayor que en aquellos que presentaron quemaduras leves. Los pacientes con quemaduras eléctricas tuvieron una estancia hospitalaria mayor en comparación con los de otras etiologías; así mismo, aquellos pacientes con una superficie corporal afectada superior al $30 \%$ permanecieron más días hospitalizados en comparación con aquellos con lesiones menos extensas, datos semejantes a los descritos. ${ }^{(15)}$

El $18.8 \%$ de los pacientes que sufrieron quemaduras por electricidad requirieron amputación de la extremidad afectada. ${ }^{(15)}$ La mortalidad descendió del $4.3 \%$ hasta el $1.1 \%$ en comparación con lo reportado hace 11 años en un estudio realizado en esta casa de salud; ${ }^{(8)}$ además, observamos que la mortalidad estuvo relacionada con pacientes que presentaron más del $50 \%$ de superficie corporal quemada con compromiso de vía aérea, de forma similar a lo reportado por otros autores. ${ }^{(15,18)}$ En lo que respecta a la etiología, observamos una relación entre mortalidad y quemaduras causadas por fuego. ${ }^{(19)}$

\section{Conclusiones}

Entre la población pediátrica atendida en el Hospital Baca Ortiz de Quito, Ecuador, observamos que los menores de 5 años son los más vulnerables a sufrir quemaduras, lo que se puede atribuir a la falta de precaución por parte de su cuidador y a la poca respuesta de los niños ante situaciones de peligro. 
La principal etiología de las quemaduras fue líquido hirviente. Los casos fatales tuvieron relación con quemaduras causadas por fuego y lesión de vía aérea.

Los pacientes con quemaduras eléctricas tuvieron una mayor estancia hospitalaria y un aumento de la necesidad de amputación de la extremidad afectada, lo que se puede atribuir a la profundidad y severidad de las injurias causadas por este medio.

La mortalidad descendió del $4.3 \%$ hasta el $1.1 \%$ en comparación con lo reportado hace 11 años en un estudio realizado en este hospital.

\section{Dirección del autor}

Dr. Pablo Gallegos Torres

Servicio de Cirugía Plástica

Hospital Baca Ortiz

Av. 6 de diciembre s/n y Av. Cristóbal Colón

CP 170523, Quito, Ecuador

Correo electrónico: pablodavid89@hotmail.com

\section{Bibliografía}

1. Mock C., Peck M., Krug E., Haberal M. Confronting the global burden of burns: A WHO plan and a challenge. Burns. 2009;35(5):615-617.

2. Kai-Yang L., Zhao-Fan X., Luo-Man Z., Yi-Tao J., Tao T., Wei W., et al. Epidemiology of Pediatric Burns Requiring Hospitalization in China: A Literature Review of Retrospective Studies. Pediatrics. 2008;122(1):132-1342.

3. Peck M. Epidemiology of burns throughout the world. Part I: Distribution and risk factors. Burns. 2011;37(7):1087-1100.

4. Sengoelge M., El-Khatib Z., Laflamme L. The global burden of child burn injuries in light of country level economic development and income inequality. Prev Med Reports. 2017;6:115-120.

5. Agbenorku P., Agbenorku M., Fiifi-Yankson PK. Pediatric burns mortality risk factors in a developing country's tertiary burns intensive care unit. Int J Burns Trauma. 2013;3(3):151158.
6. Mozingo D., Ahrenholz D., Cusick-Jost J., Dimick A., Greenhalgh D., Hickerson W., et al. Advanced Burn Life Support. $J$ Burn Care Rehabil. 1999;20(1):1-109.

7. Rojas M., Saavedra R., Vicencio P., Solís F. Cambios epidemiológicos en niños quemados, a 10 años de seguimiento. Rev Chil Pediatr. 2016;87(3):186-192.

8. Dávalos P., Dávila L., Meléndez A. Manejo de morbimortalidad del paciente pediátrico quemado en el hospital "Baca Ortiz" de Quito, Ecuador. Cir. plást. iberolatinoam. 2007;33(3):163-170.

9. Aldana M., Navarrete N. Epidemiology of a decade of Pediatric fatal burns in Colombia, South America. Burns. 2015;41(7):1587-1592.

10. Orozco M., Celis A., Méndez A., Miranda R. Perfil epidemiológico de niños con quemaduras del Hospital Civil de Guadalajara Dr. Juan I. Menchaca, 2009-2011. Bol Med Hosp Infant Mex. 2015;72(4):249-256.

11. Solís F., Domic C., Saavedra R., González A. Incidencia y prevalencia de las lesiones por quemaduras en menores de 20 años. Rev Chil Pediatr. 2014;85(6):674-681.

12. Goldman S., Aharonson L., Peleg K. Childhood burns in Israel: A 7-year epidemiological review. Burns. 2006;32(4):467-472.

13. Parbhoo A., Louw Q., Grimmer K. Burn prevention programs for children in developing countries requires urgent attention: A targeted literature review. Burns. 2010;36(2):164-175.

14. Duke J., Wood F., Semmens J., Edgar DW., Spilsbury K., Hendrie D., et al. A Study of Burn Hospitalizations for Children Younger Than 5 Years of Age: 1983-2008. Pediatrics. 2011; 1;127(4):e971 LP-e977.

15. Coban Y., Erkilic A., Analay H. Our 18-month experience at a new burn center in Gaziantep, Turkey. Ulus travma ve acil cerrahi Derg . Turkish J trauma $\{\&\}$ Emerg Surg TJTES. 2010;16(4):353-356.

16. D'Souza A., Nelson N., McKenzie L. Pediatric burn injuries treated in US emergency departments between 1990 and 2006. Pediatrics. 2009;124(5):1424-1230.

17. Lipovy B., Brychta P., Gregorová N., Jelínková Z., Rihová H., Suchánek I., et al. The epidemiology of pediatric burns undergoing intensive care in Burn Centre Brno, Czech Republic, 1997-2009. Burns. 2012;38(5):776-782.

18. Pavoni V., Gianesello L., Paparella L., Buoninsegni L., Barboni E. Outcome predictors and quality of life of severe burn patients admitted to intensive care unit. Scand J Trauma Resusc Emerg Med. 2010;18(1):24.

19. Den Hertog P., Blankendaal F., Ten Hag S. Burn injuries in The Netherlands. Accid Anal Prev. 2000;32(3):355-364. 
